\title{
Atendimentos a Crianças e Adolescentes com Câncer em Minas Gerais: Retrato de uma Década
}

doi: https://doi.org/10.32635/2176-9745.RBC.2018v64n3.42

\author{
Assistance to Children and Teenagers with Cancer in Minas Gerais: Portrait of a Decade \\ Asistencia a Niños y Adolescentes con Cáncer en Minas Gerais: Retrato de una Década
}

\section{Murilo César do Nascimento' ${ }^{1}$}

Resumo

Introduçáo: $\mathrm{O}$ câncer em crianças e adolescentes possui relevância epidemiológica em escala global. Objetivo: Conhecer e descrever a configuraçáo epidemiológica, clínica e assistencial dos atendimentos a crianças e adolescentes com câncer tratados em Minas Gerais, de 2007 a 2016. Método: Estudo exploratório, descritivo, com dados secundários via IntegradorRHC, sobre os registros de atendimentos no Estado a crianças e adolescentes com câncer. Resultados: Houve 4.953 atendimentos a crianças e adolescentes com câncer. Predominaram a faixa etária de 15 a 19 anos; indivíduos do sexo masculino; e pardos. A localização primária do tumor mais frequentes foi o sistema hematopoiético. A base principal para o diagnóstico do tumor predominante foi a histologia do tumor primário. O primeiro tratamento recebido no hospital que prevaleceu foi a quimioterapia; as razóes para não tratar mais preenchidas, depois das opções "não se aplica", "outras razóes" e "sem informação", foram o "óbito" e o "tratamento realizado fora". "Doença estável” significando "paciente estável" foi o estado da doença ao final do primeiro tratamento mais observado. A clínica no início do tratamento mais frequente foi a oncologia clínica. O resultado "leucemia linfoblástica de células precursoras" foi o mais frequente entre os tipos histológicos identificados. Constataram-se 36 instituições que compóem a rede de oncologia em 21 municípios do Estado. Verificou-se aparente redução dos registros/atendimentos a crianças e adolescentes com câncer. Conclusáo: A caracterização e a descriçáo propostas foram alcançadas e retrataram os atendimentos, em Minas Gerais, a crianças e adolescentes com câncer entre 2007 a 2016.

Palavras-chave: Neoplasias; Saúde da Criança; Saúde do Adolescente; Pesquisa sobre Serviços de Saúde; Institutos de Câncer.

\begin{abstract}
Introduction: Cancer in children and teenagers has global epidemiological relevance. Objective: To know and describe the epidemiological, clinical and care settings of the children and teenagers with cancer treated in Minas Gerais from 2007 to 2016. Method: An exploratory, descriptive study with secondary data via IntegratorRHC about the records of care in the State of children and teenagers with cancer. Results: There were 4,953 visits to children and teenagers with cancer. Predominate: age range of 15 to 19 years; male subjects; and brown. The most frequent primary site of the tumor was the hematopoietic system. The primary basis for the diagnosis of the tumor that predominated was the histology of the primary tumor. The first treatment received at the hospital that predominated was chemotherapy; the reasons for not dealing more filled after the "not applicable", "other reasons" and "no information" options were "death" and "treatment carried out". "Stable disease" meaning "stable patient" was the disease state at the end of the first most observed treatment. The most frequent clinic at the beginning of the treatment was Oncology Clinic. The result "Precursor Cell Lymphoblastic Leukemia" was the most frequent among histological types identified. We found 36 institutions that make up the oncology network in 21 municipalities of the State. There was a reduction of records / services to children and teenagers with cancer. Conclusion: The proposed characterization and description were reached and portrayed the visits in Minas Gerais, to children and teenagers with cancer between 2007 and 2016. Key words: Neoplasms; Child Health; Adolescent Health; Health Services Research; Cancer Care Facilities.
\end{abstract}

Resumen

Introducción: El cáncer en niños y adolescentes tiene relevancia epidemiológica a escala global. Objetivo: Conocer y describir la configuración epidemiológica, clínica y asistencial de los atendimientos a niños y adolescentes con cáncer tratados en Minas Gerais, de 2007 a 2016. Método: Estudio exploratorio, descriptivo, con datos secundarios vía IntegradorRHC, sobre los registros de atendimientos en la Provincia a los nińos y adolescentes con cáncer. Resultados: Hubo 4.953 atendimientos a niños y adolescentes con cáncer. Predominaron: grupo de edad de 15 a 19 ańos; individuos del sexo masculino; y pardos. Localización primaria del tumor más frecuente fue el sistema hematopoyético. La base principal para el diagnóstico del tumor que predominó fue la histología del tumor primario. El primer tratamiento recibido en el hospital que predominó fue la quimioterapia; las razones para no tratar más completadas después de las opciones "no se aplica", "otras razones" y "sin información" fueron el "óbito" y el "tratamiento realizado fuera". "Enfermedad estable" que significando "Paciente estable" fue el estado de la enfermedad al final del primer tratamiento más observado. La clínica al inicio del tratamiento más frecuente fue la oncología clínica. El resultado "Leucemia Linfoblástica de Células Precursoras" fue el más frecuente entre los tipos histológicos identificados. Se constataron 36 instituciones que componen la red de oncología en 21 municipios de la Provincia. Se verificó reducción de los registros / atendimientos a niños y adolescentes con cáncer. Conclusión: La caracterización y la descripción propuestas fueron alcanzadas y retrataron los atendimientos, en Minas Gerais, a los niños y adolescentes con cáncer entre 2007 a 2016.

Palabras clave: Neoplasias; Salud del Niño; Salud del Adolescente; Investigación en Servicios de Salud; Instituciones Oncológicas.

1 Universidade Federal de Alfenas (Unifal). Alfenas (MG), Brasil. Orcid iD: https://orcid.org/0000-0002-3436-2654

Endereço para correspondência: Murilo César do Nascimento. Rua Gabriel Monteiro da Silva, 700 - Centro. Alfenas (MG), Brasil. CEP $37130-001$.

E-mail: murilo@unifal-mg.edu.br. 


\section{INTRODUÇÃO}

O câncer tem se consolidado como importante problema de saúde pública em todas as regióes do mundo $^{1}$. Na escala global, mais de 150 mil crianças são diagnosticadas com câncer a cada ano ${ }^{2}$. De forma similar aos países desenvolvidos, no Brasil, o câncer representa a primeira causa de óbito por doença, entre as crianças e adolescentes ${ }^{3}$. O Estado de Minas Gerais contribuiu com aproximadamente $24 \%$ do total de casos hospitalares registrados na alta complexidade brasileira no ano de 2009. Nessa Unidade Federativa, observou-se que grande parte dos casos eram passíveis de medidas de controle e detecção que, se implementadas precocemente, poderiam contribuir de forma expressiva para uma evolução sem óbito ${ }^{4}$.

Não bastasse esse cenário epidemiológico já preocupante, estima-se que a incidência de câncer se acentuará ainda mais no futuro, gerando impacto expressivo na carga global de doenças, na qualidade de vida das pessoas acometidas e comprometendo os já limitados recursos para a assistência à saúde 5 . Considerando que a maioria das crianças e adolescentes sobreviventes de câncer requer monitoramento cuidadoso por especialistas ao longo da vida ${ }^{6}$, a análise minuciosa dos dados pessoais, clínicos e assistenciais em oncologia pediátrica pode ajudar os gestores de saúde pública a atenderem melhor as necessidades de cuidados crônicos para crianças e adolescentes ${ }^{7}$.

No Brasil, o Instituto Nacional de Câncer José Alencar Gomes da Silva (INCA) é o órgão da administração direta do Ministério da Saúde, com capilaridade crescente em todos Estados, que tem como missão o desenvolvimento e a coordenação das açôes integradas para a prevenção e o controle do câncer no país. Podem ser citadas como açóes do INCA a assistência multiprofissional, prestada direta e gratuitamente pelo Sistema Único de Saúde (SUS); a atuação em áreas estratégicas, como prevençáo e detecção precoce; a formação de profissionais especializados nas diversas áreas da saúde; o desenvolvimento da pesquisa; e a geração de informação epidemiológica ${ }^{8}$.

Uma fonte de dados transversal a todas essas açóes de pesquisa e enfrentamento do câncer, desde a escala locorregional até o cenário nacional, é constituída pelos Registros de Câncer. São eles os centros que operam a coleta, o armazenamento, o processamento e a análise de informaçóes sobre pacientes ou pessoas com diagnóstico confirmado de câncer. Tais centros podem ser de base populacional (Registros de Câncer de Base Populacional RCBP) ou de base hospitalar (Registros de Câncer de Base Hospitalar - RHC) e figuram-se extremante relevantes para estudos epidemiológicos e para o detalhamento de aspectos pessoais, clínicos e assistenciais da oncologia no Brasil ${ }^{9}$.

Particularmente, os RHC têm como finalidade contribuir para a melhoria da assistência prestada ao paciente e para o planejamento intrainstitucional, para auxiliar na organização de um Sistema de Informaçóes em Câncer e para promover educação profissional continuada aos profissionais da área de saúde coletiva. Ademais, as informaçôes produzidas em um RHC também refletem o desempenho do corpo clínico na assistência prestada ao paciente .

Diante do exposto e considerando a magnitude do problema do câncer na atualidade, que, para além da abordagem do cuidado em saúde, apresenta facetas sociais e econômicas importantes ${ }^{1,2,9}$, é que se justifica a necessidade de adensar o conhecimento científico, a vigilância em saúde e o enfrentamento do câncer em crianças e adolescentes.

Dessa forma, o objetivo do presente trabalho foi conhecer e descrever a configuração epidemiológica, clínica e assistencial dos atendimentos a crianças e adolescentes com câncer tratadas no Estado brasileiro de Minas Gerais, entre os anos de 2007 a 2016. Para tal, procurou-se caracterizar demográfica e clinicamente as crianças e adolescentes que passaram por atendimento oncológico no período; identificar os municípios que compóem a rede assistencial e de registro hospitalar de câncer no Estado; e descrever a distribuição temporal dos atendimentos em oncologia a crianças e adolescentes.

\section{MÉTODO}

Trata-se de um estudo exploratório, descritivo, realizado com dados secundários de domínio público sobre os atendimentos da alta complexidade oncológica no Estado de Minas Gerais. Dessa forma, o público-alvo da pesquisa foi composto por todos os registros hospitalares de câncer do Estado de Minas Gerais, com bases de dados disponíveis para o período de 2006 a 2017, referentes à idade de 0 a 19 anos, por se tratarem do recorte espacial, temporal e etário de interesse.

Os dados dos "casos analíticos" e dos "casos não analíticos" foram obtidos sem distinção por meio de consulta eletrônica ao "Tabulador Hospitalar" IntegradorRHC do Sistema de Informação de Registro Hospitalar de Câncer (SisRHC), a partir da seleção da base estadual de Minas Gerais que, como as demais, é disponibilizada on-line e possui acesso irrestrito a usuários e ao público geral ${ }^{10}$. O pesquisador utilizou um notebook de uso institucional com acesso à internet na própria Universidade de origem para proceder à coleta, tabulação e análise dos dados. 
As variáveis selecionadas relacionadas às pessoas foram: sexo; faixa etária; e raça/cor. Sobre os aspectos clínicos/ assistenciais, trabalharam-se as variáveis localização primária do tumor por grupo, base mais importante para o diagnóstico do tumor, primeiro tratamento recebido no hospital, principal razão para a não realização do tratamento antineoplásico no hospital, estado da doença ao final do primeiro tratamento no hospital, clínica de início do tratamento e tipo histológico do tumor primário. Já em relaçáo ao tempo, atentou-se para o campo ano da primeira consulta; e, no que se refere ao lugar foi priorizado, no tabulador, o município da unidade hospitalar.

Os dados tabulares e cartográficos foram organizados e analisados com o auxílio dos Softwares Stata 11.0, Epi Info 7, Excel 2013 e TabWin 4.1.5. As variáveis foram analisadas por meio de estatística descritiva, sendo que os dados nominais e categóricos foram submetidos à análise e à apresentação da frequência simples e relativa, os dados contínuos foram analisados e apresentados segundo as medidas de tendência central média, mediana e moda, bem como as medidas de dispersão variância e desvio-padrão.

Em atenção às diretrizes e normas regulamentadoras de pesquisas envolvendo seres humanos, das quais trata a Resolução n ${ }^{\circ}$. 466, de 12 de dezembro de $2012^{11}$, esclarece-se que, em razão do método utilizado neste trabalho - no que se refere ao delineamento, ao público-alvo e à natureza da pesquisa, bem como considerando o tipo, a fonte, a técnica de coleta e a análise dos dados - não houve necessidade de submissão de projeto para apreciação pelo Comitê de Ética em Pesquisa envolvendo Seres Humanos.

\section{RESULTADOS}

Foram registrados 4.953 atendimentos a crianças e adolescentes no Estado de Minas Gerais, entre os anos de 2007 e 2016. Em relação às características demográficas dessa população com idade entre 0 a 19 anos, a faixa etária mais frequente foi a de 15 a 19 anos $(1.744 ; 35,21 \%)$, seguida pelas faixas de 0 a 4 anos (1.244; 25,12\%), 10 a 14 anos (1.095; 22,11\%), e 5 a 9 anos de idade, com 870 registros, o equivalente a $17,56 \%$ dos registros. Observou-se que a média das idades foi de 10,49 anos, que a mediana e a moda equivaleram há 12 anos, com variância de 37,93 e desvio-padrão de $\pm 6,15$.

Verificou-se que 2.645 registros se referiram a indivíduos do sexo masculino $(53,40 \%)$ e 2.308 informaçóes originaram-se de pessoas do sexo feminino $(46,60 \%)$. No que se refere à raça/cor dos atendidos, registrou-se a seguinte distribuição: 2.130 pardos (43,00\%), 1.792 brancos (36,18\%), 409 pretos $(8,26 \%)$, 19 amarelos $(0,38 \%)$ e 8 indígenas $(0,16 \%)$; ainda sobre essa variável, foram encontrados 595 campos "Sem Informação", o que correspondeu a $12,01 \%$ dos registros.

Uma das características clínicas importantes sobre as crianças e adolescentes com câncer diz respeito às localizaçôes primárias dos tumores. Convém lembrar que os Registros Hospitalares de Câncer utilizam uma adaptação da Classificaçáo Internacional de Doenças para Oncologia, a CID-O ${ }^{12}$, para codificar os tumores, classificando-os segundo à topografia e à sua histologia. A distribuição das localizaçóes de acordo com os referidos grupos é apresentada no Gráfico 1, segundo a frequência relativa das crianças e os percentuais por faixas etárias.

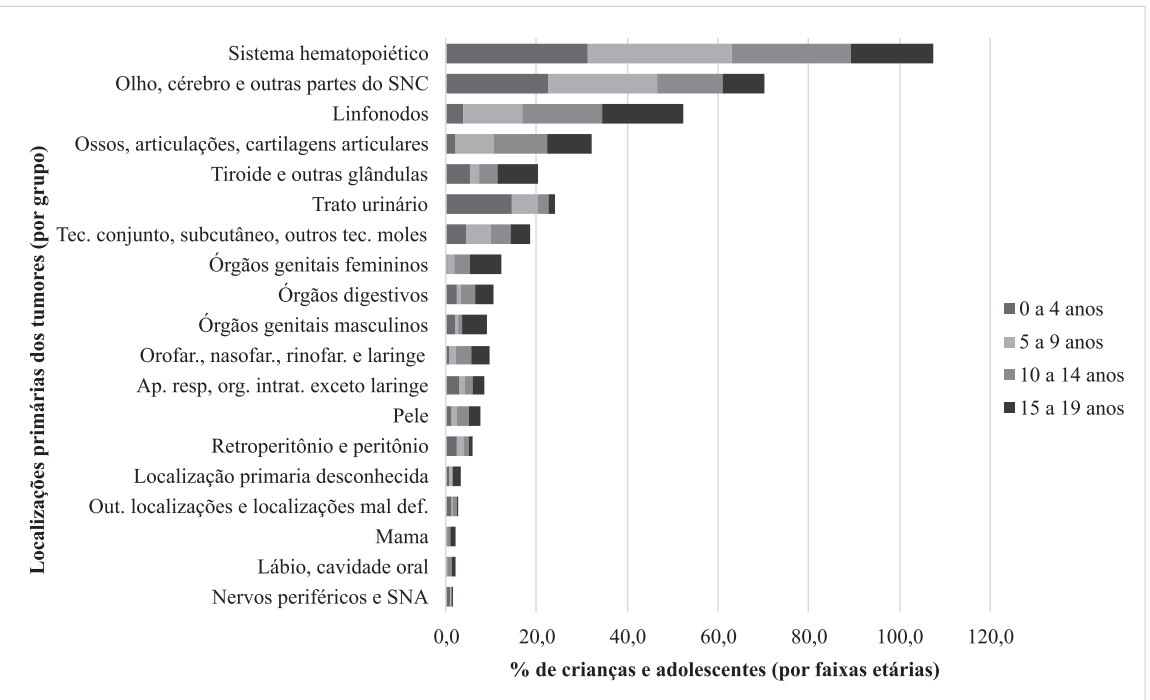

Gráfico 1. Distribuição percentual da localização primária dos tumores, por grupo da CID-O, segundo a faixa etária das crianças e adolescentes, atendidos no Estado de Minas Gerais, entre 2007 e 2016

Fonte: INCA ${ }^{10}$. 
Em relação aos outros aspectos clínicos e assistenciais investigados, o detalhamento das bases para o diagnóstico do tumor, das principais razóes para não tratar no hospital, dos primeiros tratamentos recebidos, assim como o estado da doença ao final do primeiro tratamento no hospital podem ser visualizados na Tabela 1 .

Entre as clínicas observadas no início do tratamento oncológico, as dez mais frequentes foram: Oncologia Clínica (1.282 casos; 25,88\%), Pediatria Oncológica (730 casos; 14,74\%), Hematologia Clínica $(698$ casos; 14,09\%), Radioterapia (662 casos; 13,37\%), Neurocirurgia (212 casos; 4,28\%), Oncologia Cirúrgica (186 casos; 3,76\%), Pediatria Cirúrgica (146 casos; 2,95\%), Cabeça e Pescoço (117 casos; 2,36\%), Cirurgia Geral (88 casos; $1,78 \%$ ) e Ortopedia, com 74 casos, correspondentes a $1,49 \%$ dos registros. Já as clínicas de Ginecologia, Oftalmologia, Urologia, Pediatria, Medicina Nuclear, Cirurgia Plástica, Cirurgia Torácica, Neurologia, Dermatologia e Outras totalizaram 6,10\% dos casos com 303 registros. Em quatro atendimentos, essa informação não foi registrada $(0,08 \%)$ e, em 451 registros, constou a opção "não se aplica" (9,11\% dos casos).

Já os resultados quanto ao tipo histológico mais frequente na população infantojuvenil foram apresentados conforme raça/cor e segundo as três localizaçôes primárias dos tumores mais registradas no Estado: "Sistema hematopoiético", "Olho, cérebro e outras partes do SNC" e "Linfonodos", conforme a CID-O. Por causa da quantidade elevada de classificaçôes histológicas, optou-se por apresentar os três tipos histológicos mais frequentes para cada uma das principais localizaçóes primárias do tumor. Dessa forma, é representada no Gráfico 2 a distribuição percentual de nove tipos histológicos mais frequentes, segundo a raça/cor e as principais localizaçóes primárias de tumor. Para efeito de melhor visualização, na imagem síntese, não foram apresentados os registros que indispunham da informaçáo de raça/cor, referentes a cada tipo histológico (4,97\% do total de registros).

Verificou-se que a rede assistencial da alta complexidade oncológica mineira é formada por 36 instituiçôes que ofertam atendimentos oncológicos e dispóem de Unidades Notificadoras para alimentação do SisRHC no Estado. Esses estabelecimentos estão distribuídos por 21 municípios, sendo eles: Alfenas, Barbacena, Belo Horizonte, Betim, Cataguases, Curvelo, Divinópolis, Governador Valadares, Ipatinga, Juiz de Fora, Montes Claros, Muriaé, Passos, Poços de Caldas, Ponte Nova, Pouso Alegre, São João del Rei, Sete Lagoas, Uberaba, Uberlândia e Varginha.

$\mathrm{Na}$ Capital do Estado, Belo Horizonte, foram realizados 2.794 atendimentos, que corresponderam a $56,41 \%$ do total de registros. Os municípios de Montes

Tabela 1. Aspectos clínicos e assistenciais de crianças e adolescentes com câncer atendidos em Minas Gerais de 2007 a 2016

\begin{tabular}{|c|c|c|c|}
\hline Variável & Domínio & $\mathbf{N}$ & $\%$ \\
\hline \multirow{8}{*}{$\begin{array}{l}\text { Base mais importante para } \\
\text { o diagnóstico do tumor }\end{array}$} & Histologia do tumor primário & 3.517 & 71,01 \\
\hline & Exame por imagem & 314 & 6,34 \\
\hline & Marcadores tumorais & 223 & 4,50 \\
\hline & Citologia & 697 & 14,07 \\
\hline & Clínica & 97 & 1,96 \\
\hline & Histologia da metástase & 41 & 0,83 \\
\hline & Pesquisa clínica & 34 & 0,69 \\
\hline & Sem informação & 30 & 0,61 \\
\hline \multirow{6}{*}{$\begin{array}{l}\text { Primeiro tratamento } \\
\text { recebido no hospital }\end{array}$} & Quimioterapia & 2.108 & 42,56 \\
\hline & Cirurgia & 657 & 13,26 \\
\hline & Radioterapia & 614 & 12,40 \\
\hline & Nenhum & 499 & 10,07 \\
\hline & Vários tratamentos menos frequentes* e outros & 1.071 & 21,62 \\
\hline & Sem informação & 4 & 0,08 \\
\hline \multirow{8}{*}{$\begin{array}{l}\text { Estado da doença ao final } \\
\text { do primeiro tratamento no } \\
\text { hospital }\end{array}$} & Doença estável (paciente estável) & 1.378 & 27,82 \\
\hline & Sem evidência da doença (remissão completa) & 572 & 11,55 \\
\hline & Não se aplica & 569 & 11,49 \\
\hline & Óbito & 441 & 8,90 \\
\hline & Remissão parcial & 385 & 7,77 \\
\hline & Doença em progressão & 332 & 6,70 \\
\hline & Suporte terapêutico oncológico & 30 & 0,61 \\
\hline & Sem informação & 1.246 & 25,16 \\
\hline
\end{tabular}

Legenda: *Tratamentos menos frequentes (siglas): Cir + Qt; Qt + Rxt; Cir + Qt + Rxt; Cir + Rxt; Outros + Qt; Ht + Qt; Ht; Outros + Qt + Rxt; Qt + TMO; Cir + Outros; TMO; Cir + Outros + Qt; Cir + Ht; Ht + Rxt; Cir + Ht + Qt; Cir + Outros + Qt + Rxt; Ht + Outros + Qt + Rxt. 


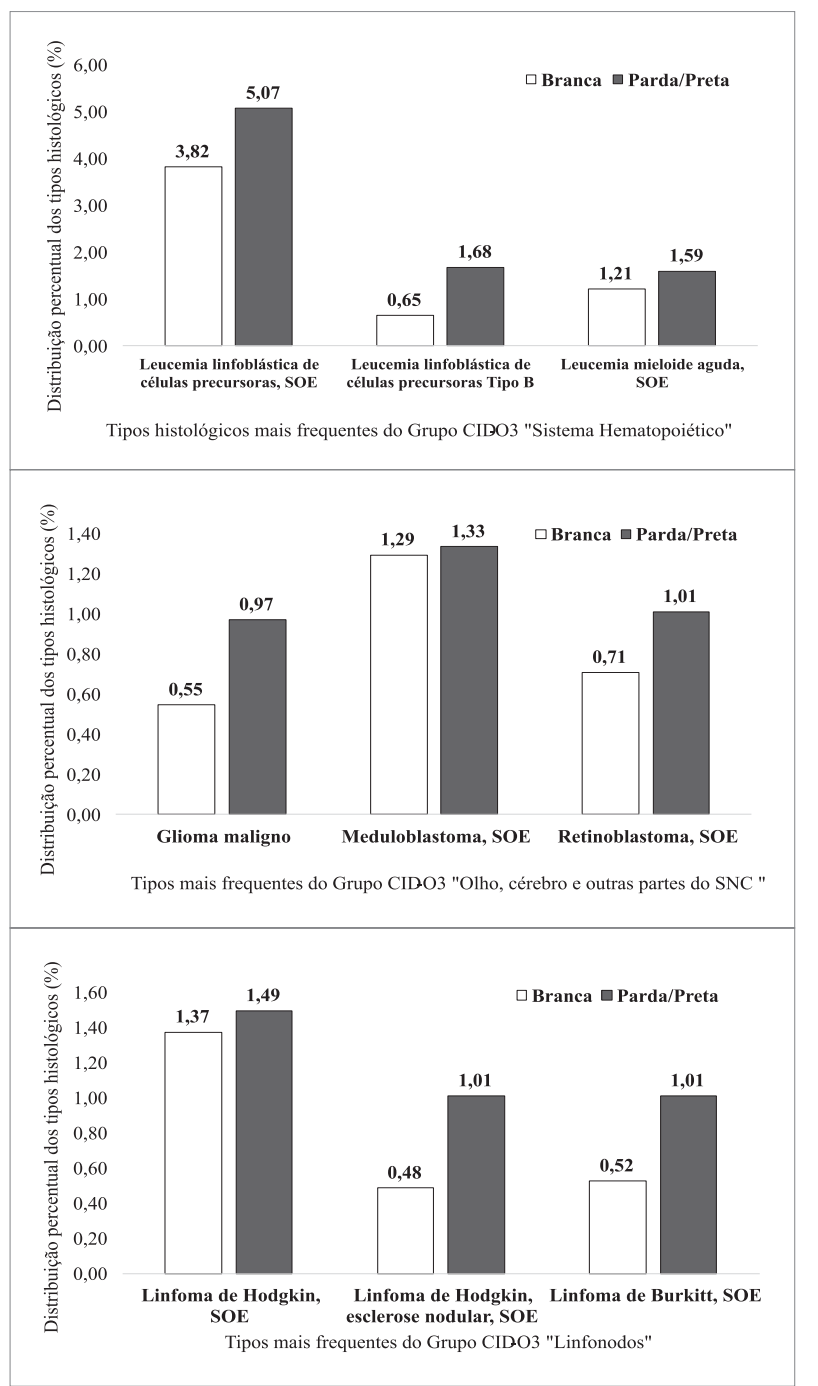

Gráfico 2. Distribuição percentual dos tipos histológicos mais frequentes, segundo as principais localizações primárias dos tumores e conforme a raça/cor mais frequente das crianças e dos adolescentes atendidos por câncer em Minas Gerais, entre 2007 e 2016 Fonte: $\mathrm{INCA}^{10}$.
Claros e Juiz de Fora se destacaram em segundo e terceiro lugar ao computarem 391 registros $(7,89 \%)$ e 363 registros $(7,33 \%)$ entre o somatório de atendimentos em oncologia para a referida faixa etária. A distribuição espacial do número de atendimentos a crianças e adolescentes com câncer pelos municípios mineiros, no recorte de tempo analisado, é representada pelo mapa temático da Figura 1.

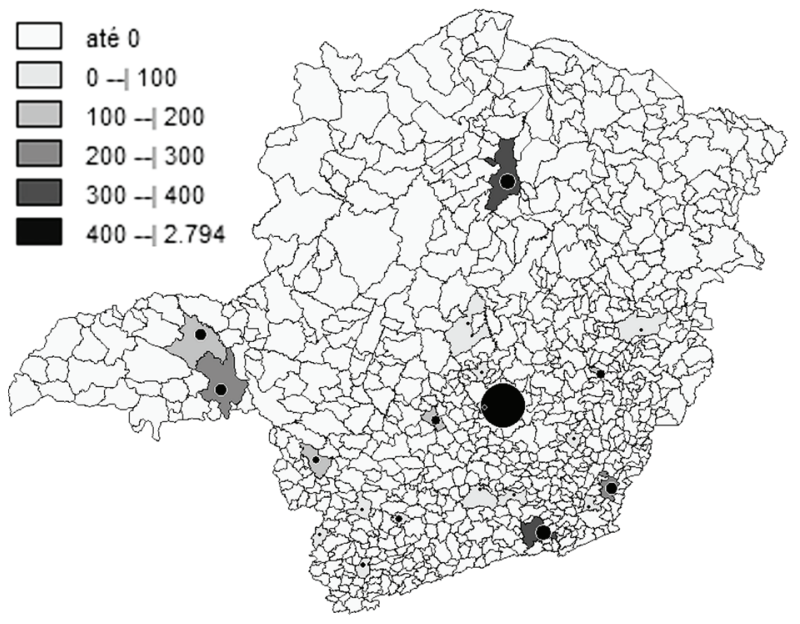

Figura 1. Distribuição espacial do número de atendimentos a crianças e adolescentes com câncer pelos municípios mineiros com Unidades Hospitalares Notificadoras, entre 2007 e 2016 Fonte: $\mathrm{INCA}^{10}$.

Já a distribuição temporal das crianças e dos adolescentes com câncer, atendidos no Estado de Minas Gerais, entre os anos de 2007 e 2016, pode ser visualizada no Gráfico 3.

Em relação à série histórica de dez anos analisada, os registros foram mais frequentes nos anos de 2007 $(589 ; 11,89 \%)$ e 2008 (563; 11,37\%), com aparente

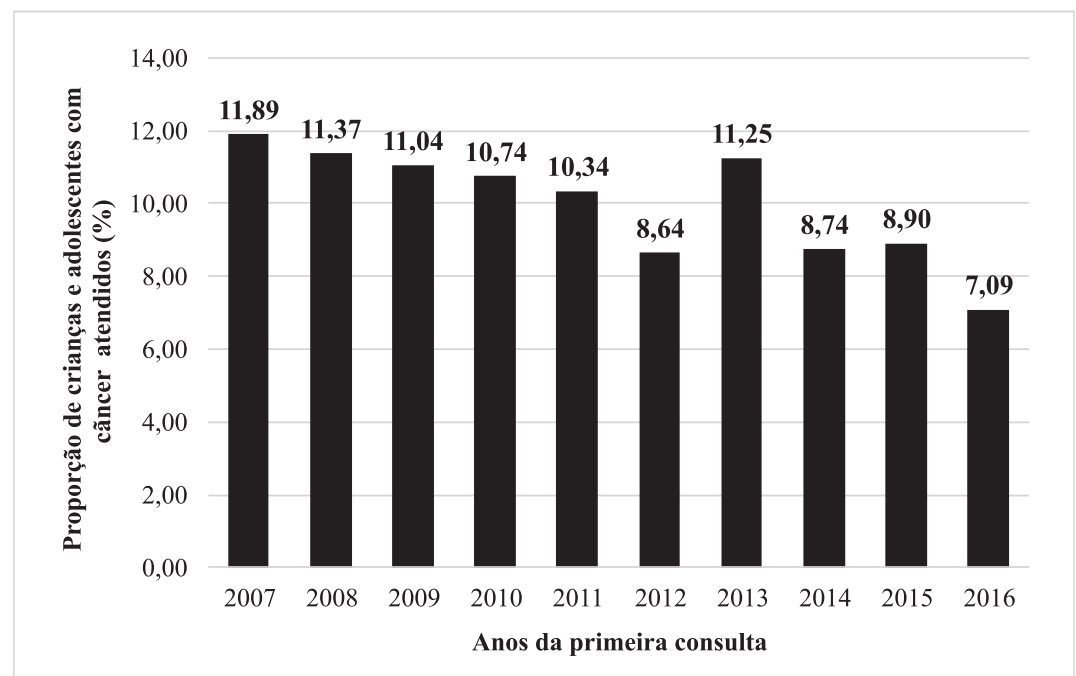

Gráfico 3. Distribuição temporal das crianças e dos adolescentes com câncer, atendidos no Estado de Minas Gerais, entre os anos de 2007 e 2016

Fonte: $\mathrm{INCA}^{10}$. 
oscilaçáa ao longo do tempo, como pode ser visto no total de registros para o ano de 2016, que apresentou 351 atendimentos $(7,09 \%)$.

\section{DISCUSSÃO}

O câncer infantojuvenil no Brasil corresponde a 3\% dos casos no país, acometendo aproximadamente 12.500 casos novos de crianças e adolescentes (até os 19 anos) $)^{9}$. Apesar da expansáo do número de serviços habilitados em alta complexidade oncológica pós-criação do SUS, essa rede assistencial ainda se configura insuficiente frente às demandas epidemiológicas ${ }^{13}$. Nesse cenário, nem toda a população que necessita é acolhida em serviços qualificados, e determinadas barreiras ao acesso podem gerar atraso no diagnóstico, agravamento do estado de saúde, e até mesmo óbito dessas crianças e adolescentes ${ }^{14}$. Por isso, reforça-se a importância de conhecer o perfil de atendimentos a crianças e adolescentes com câncer.

Em relação ao acometimento infantojuvenil, para muitos tipos de câncer, existem diferenças relativas à sobrevivência, que podem ser atribuídas a fatores socioeconômicos subjacentes. Há na literatura registro de associação entre raça/etnia e sobrevivência para leucemia linfoblástica aguda, leucemia mieloide aguda, neuroblastoma e linfoma não Hodgkin, por exemplo ${ }^{15}$. Os tipos de câncer mais comuns nas crianças e adolescentes são as leucemias, os tumores do sistema nervoso central e os linfomas ${ }^{9}$. Sobre as localizaçôes primárias dos tumores por grupos das crianças e adolescentes atendidos em Minas Gerais, as mais frequentes foram o "Sistema hematopoiético", "Olho, cérebro e outras partes do SNC" e "Linfonodos".

Em um estudo sobre morbidade hospitalar, realizado pela INCA, em âmbito nacional, no ano de 2016, as maiores frequências foram observadas para os carcinomas (com exceção de pele), que representaram 38,4\% dos casos, seguidos por linfomas, com $18,3 \%$, e leucemias, com $10,9 \%$. Juntas, essas três neoplasias somaram $67,6 \%$ do total de casos de câncer nos adolescentes e adultos jovens ${ }^{9}$.

No que se refere à base mais importante para o diagnóstico do tumor apresentada na Tabela 1, observa-se que a "histologia do tumor primário" predominou com mais de $70 \%$ dos registros. Este é um dado expressivo, uma vez que existem diferenças importantes entre os tipos histológicos que geram diferenças no perfil epidemiológico da incidência e dos picos etários?.

Conhecer as razóes para não tratar e o estado da doença ao final do primeiro tratamento é essencial para compreender a prognose, bem como para avaliar a efetividade e o tipo do tratamento realizado/necessário às crianças e adolescentes com câncer. Assim, os dados trazidos por este estudo sobre os primeiros tratamentos recebidos, sobre as principais razōes para não tratar no hospital e sobre estado da doença ao final do primeiro tratamento no hospital configuram aspectos relevantes para o acompanhamento longitudinal dessa população.

As razóes para não tratar mais preenchidas depois das opçôes "não se aplica", "outras razôes" e "sem informação" foram o "óbito" e o "tratamento realizado fora". Em relaçáo à categoria mais frequente de razóes para náo tratar ter sido a opção "Não se aplica", convém esclarecer que a distribuição acentuada de tal dado é justificada pelo fato de a maioria dos pacientes ter recebido o tratamento. Nesse contexto, não se tratou de um problema de registro, mas sim de um aspecto positivo em relação à prestação da assistência.

Apesar de mais de $80 \%$ das crianças diagnosticadas com câncer apresentarem cura, a crescente população de sobreviventes de câncer infantil experimenta altas taxas de morbidade e mortalidade por conta dos "efeitos tardios" do tratamento. Tanto que diretrizes internacionais foram desenvolvidas para padronizar a triagem de sobreviventes, abordando vários modelos de cuidados de sobrevivência. Segundo tais recomendaçóes, o modelo ideal depende de diversos fatores individuais, que incluem as necessidades e preferências da pessoa, bem como os recursos locais com uma transição bem coordenada de atendimento entre os serviços ${ }^{6}$.

Ao identificar os municípios que compóem a rede assistencial e de registro hospitalar de câncer no Estado, notou-se que, dos 21 municípios com Unidade Notificadoras, 15 correspondem a localidades onde se situam Superintendências Regionais de Saúde (SRS) ou Gerências Regionais de Saúde (GRS). Os demais municípios que não são polo nem sede de Micro e Macrorregiáo de Saúde constituem-se representantes das suas respectivas Regiōes de Saúde ${ }^{16}$, o que é positivo do ponto de vista estratégico. Tal distribuição espacial traduz um alinhamento entre o nível de atenção à saúde demandado pela alta complexidade oncológica e o princípio operativo do SUS da regionalização, o que corrobora a economia de escopo e de escala em saúde.

Por fim, comparando a distribuiçáo temporal dos atendimentos pelos dez anos analisados, observa-se uma oscilação entre os registros do primeiro ao último ano da série histórica. Em razão da natureza descritiva deste trabalho, não é possível estabelecer relaçôes causais sobre a aparente redução do número de atendimentos. No entanto, se for considerado que há unidades hospitalares com participação importante nos registros do SisRHC que não apresentaram casos em 2015 e 2016, acredita-se que a simples ausência do registro possa explicar a aparente redução no número de atendimentos. Se tal 
inferência for verdadeira, depara-se com um importante sinal sobre a importância de se atentar e investir no fluxo/ encaminhamento apropriados dos registros.

Outra hipótese é de que um adensamento tecnológico na alta complexidade oncológica do Estado tenha interferido de forma positiva na distribuição dos registros. Tal possibilidade faz sentido se for considerada, por exemplo, uma consequente reduçáo de eventuais encaminhamentos entre instituiçôes para complementaçóes terapêuticas com recursos distintos. Se a hipótese do aprimoramento dos recursos terapêuticos estiver correta, a aparente redução do número de casos terá outro significado. Pacientes que antes seriam registrados mais de uma vez (em mais de uma instituição), com a extinta necessidade de encaminhamento, passariam a constar no Sistema como um registro único. Em vez da conotação negativa de uma possível diminuição no número de atendimentos, a interpretação teria a ver com o impacto positivo de uma melhor estruturação dos serviços.

Como optou-se nesta pesquisa por trabalhar tanto com dados de "casos analíticos" como de "casos não analíticos", é possível que determinados pacientes tenham sido registrados mais de uma vez, em diferentes instituiçôes, no decorrer do recorte temporal abordado. Acredita-se que, se a escolha inicial tivesse sido de se trabalhar apenas com dados de casos analíticos, ter-se-ia mais clareza sobre a série histórica dos atendimentos a crianças e adolescentes com câncer no Estado. Por se reconhecer essa limitaçáo de estudo, sugere-se que outras pesquisas complementares sejam desenvolvidas, a fim de identificar fatores explicativos para a variação temporal na distribuição dos registros.

Há na literatura menção sobre a completude e a consistência dos registros de câncer ${ }^{17}$, na qual se apontou para a importância e a necessidade de melhoria dos registros médicos, com a estruturação de campos obrigatórios e a padronização do sistema de prontuários ${ }^{18}$. Por isso, mesmo observando que o Estado de Minas Gerais possui boa completude das variáveis referentes aos aspectos clínico-epidemiológicos, o que merece destaque positivo no contexto da vigilância e do controle do câncer infantojuvenil, reforça-se aqui a necessidade de se manter/ aprimorar, de forma contínua e sistemática, a qualidade do preenchimento/alimentação do SisRHC.

Apesar de os dados produzidos nas Unidades de Registros Hospitalares de Câncer não serem utilizados para cálculo de incidência, uma vez que retratam a caracterizaçáo dos atendimentos de uma determinada instituiçáo ou conjunto de estabelecimentos ${ }^{3,10}$, acredita-se que o perfil dos atendidos e dos atendimentos, entre 2007 e 2016, possa constituir um sinalizador importante da demanda e do fluxo referente à rede de serviços da alta complexidade em oncologia que atuam no controle do câncer em crianças e adolescentes no Estado de Minas Gerais.

\section{CONCLUSÃO}

Este trabalho possibilitou conhecer e descrever a configuração epidemiológica, clínica e assistencial dos atendimentos às crianças e adolescentes com câncer tratadas em Minas Gerais, entre 2007 a 2016. Explorar as informações dos registros hospitalares de câncer permitiu realizar uma caracterização demográfica e clínica das crianças e adolescentes que passaram por atendimento oncológico; identificar os municípios que compóem a rede assistencial em oncologia com registros hospitalares de câncer; e descrever a distribuição temporal dos atendimentos em oncologia a crianças e adolescentes. Espera-se que os resultados deste estudo venham adensar o conhecimento científico sobre o tema, bem como contribuir, corroborando informaçóes epidemiológicas úteis para a vigilância em saúde e para o aprimoramento do controle do câncer em crianças e adolescentes no Estado.

\section{AGRADECIMENTOS}

A todos os que se dedicam aos Registros Hospitalares de Câncer no Estado de Minas Gerais, Brasil.

\section{DECLARAÇÃO DE CONFLITO DE INTERESSES}

Nada a declarar.

\section{FONTES DE FINANCIAMENTO}

Não há.

\section{REFERÊNCIAS}

1. Siegel RL, Miller KD, Jemal A. Cancer statistics, 2018. CA Cancer J Clin [Internet]. 2018 Jan [cited 2018 Jul 11];68(1):7-30. Available from: https://onlinelibrary. wiley.com/doi/pdf/10.3322/caac.21442

2. Sociedade Brasileira de Pediatria, Departamento Científico de Oncologia. Atuação do pediatra: epidemiologia e diagnóstico precoce do câncer pediátrico. [Documento Científico]. Soc Bras Pediatr [Internet]. 2017;1:1-7. [acesso 2018 Jul 11]. Disponível em: http://www.sbp.com.br/fileadmin/user_upload/ publicacoes/C-Doc-Cientifico-Oncologia-Epidemiol30-mar-17.pdf

3. Instituto Nacional de Câncer José Alencar Gomes da Silva. Incidência, mortalidade e morbidade hospitalar por câncer em crianças, adolescentes e adultos jovens no 
Brasil: informaçóes dos registros de câncer e do sistema de mortalidade. [Internet]. Rio de Janeiro: INCA; 2016. 412 p. [acesso 20198 Jul 11]. Disponível em: http://www1.inca. gov.br/wcm/incidencia/2017/pdf/versao-completa.pdf

4. Secretaria de Estado de Saúde de Minas Gerais (BR). Situação do câncer em Minas Gerais e suas macrorregióes de saúde: estimativas de incidência e mortalidade para o ano 2013, válidas para 2014: perfil da mortalidade: perfil da assistência na alta complexidade. Vol. 1 [Internet]. Belo Horizonte: SES-MG; 2013. [acesso 2018 Jul 11]. Disponível em: https://bvssp.icict.fiocruz.br/lildbi/ docsonline/get.php?id=4181

5. Global Burden of Disease Cancer Collaboration; Fitzmaurice C, Allen C, Barber RM, Barregard L, Bhutta ZA, et al. Global, regional, and national cancer incidence, mortality, years of life lost, years lived with disability, and disability-adjusted life-years for 32 cancer groups, 1990 to 2015. JAMA Oncol [Internet]. 2017 Apr [cited 2018 Jul 12]; 3(4):524-548. Available from: https://jamanetwork.com/journals/jamaoncology/ fullarticle/2588797

6. Song A, Fish JD. Caring for survivors of childhood cancer. Curr Opin Pediatr. 2018 Dec;30(6):864-873.

7. Siegel DA, Li J, Henley SJ, Wilson RJ, Lunsford $\mathrm{NB}$, Tai E, et al. Geographic variation in pediatric cancer incidence United States, 2003-2014. Morb Mor Wkly Rep [Internet]. 2018 Jun 29[cited 2018 Jul 12];67(25):707-713. Available from: http://www. cdc.gov/mmwr/volumes/67/wr/mm6725a2.htm?s_ $\mathrm{cid}=\mathrm{mm} 6725 \mathrm{a} 2 \_\mathrm{w}$

8. Ministério da Saúde (BR), Instituto Nacional de Câncer José Alencar Gomes da Silva Glossário temático: controle de câncer [Internet]. Brasília: Ministério da Saúde; 2013. [acesso 2018 Jul 13]. Disponível em: http://bvsms.saude. gov.br/bvs/publicacoes/glossario_tematico_controle_ cancer.pdf

9. Instituto Nacional de Câncer José Alencar Gomes da Silva. Estimativa 2018: Incidência do câncer no Brasil [Internet]. Rio de Janeiro: INCA; 2017. [acesso 2018 Jul 16]. Disponível em: http://www1.inca.gov.br/ estimativa/2018/estimativa-2018.pdf

10. Instituto Nacional de Câncer José Alencar Gomes da Silva. IntegradorRHC. [Internet]. Versão 3.2. Rio de Janeiro: INCA [2014] - [atualizado 2018 Jul 6; acesso 2018 Jul 15]. Disponível em: https://irhc.inca.gov.br/ RHCNet/

11. Ministério da Saúde (BR), Conselho Nacional de Saúde. Resolução $\mathrm{n}^{\circ} 466$, de 12 de dezembro de 2012 [Internet]. [acesso 2018 Jul 17]. Disponível em: http://bvsms.saude.gov.br/bvs/saudelegis/cns/2013/ res0466_12_12_2012.html

12. Rebelo PA, Paiva Filho FS, Sousa AF, editores. Comentários sobre a "Classificação Internacional de Doenças para Oncologia - segunda edição (Cid-O/2)”. In: Rebelo PA,
Paiva Filho FS, Sousa AF, editores. Registro hospitalar de câncer: dados dos hospitais do INCA, relatório anual 1994/1998. [Internet]. Rio de Janeiro: INCA; 2004 [acesso 2019 Jan 03]. Anexo. Disponível em: www1.inca. gov.br/rhc/docs/anexo_coment2edicao.pdf

13. Silva MJ, Lima FL, O’Dwyer G,Castro CG. Política de atenção ao câncer no Brasil após a Criação do Sistema Único de Saúde. Rev Bras Cancerol [Internet]. 2017 [acesso 2018 Jul 17];63(3):177-187. Disponível em: http://www.inca.gov.br/rbc/n_63/v03/pdf/03-artigopolitica-de-atencao-ao-cancer-no-brasil-apos-a-criacaodo-sistema-unico-de-saude.pdf

14. Rezende AM, Santos PP, Cerqueira AC, Viana JL, Modena CM; Associação Unificada de Recuperação e Apoio. A criança e o adolescente com câncer em Casa de Apoio: projetando vivências. Rev SBP [Internet]. 2013 [acesso 2018 Ago 2];16(1):3-32. Disponível em: http://pepsic. bvsalud.org/scielo.php?script=sci_arttext\&pid=S151608582013000100002\&lng=pt

15. Kehm RD, Spector LG, Poynter JN, Vock DM, Altekruse SF, Osypuk TL. Does socioeconomic status account for racial and ethnic disparities in childhood cancer survival? Cancer [Internet]. 2018 Aug 28-Oct 15 [cited 2018 Ago 2]; 124(20):4090-4097. Available from: http://doi.wiley. com/10.1002/cncr.31560

16. Belo Horizonte (MG), Secretaria de Estado de Saúde de Minas Gerais. Municípios de Minas Gerais, Superintendências ou Gerências Regionais de Saúde [Internet]. Belo Horizonde: SES; 2011. [atualizado 2013 Nov 20; acesso 2018 Aug 22]. Disponível em: http:// www.saude.mg.gov.br/cer/page/208-municipios-deminas-gerais---superintendencias-ou-gerencias-regionaisde-saude-sesmg

17. Pinto IV, Ramos DN, Costa MC, Ferreira CB, Rebelo MS. Completude e consistência dos dados dos registros hospitalares de câncer no Brasil. Cad Saúde Colet [Internet]. 2012 [acesso 2018 Ago 20]; 20(1):113-120. Disponível em: http://www.cadernos.iesc.ufrj.br/cadernos/ images/csc/2012_1/artigos/CSC_v20n1_113-120.pdf

18. Luz CM, Deitos J, Siqueira TC, Heck AP. Completude das informaçóes do registro hospitalar de câncer em um hospital de Florianópolis. ABCS Health Sci [Internet]. 2017[acesso 2018 Ago 21];42(2):73-79. Disponível em: https://www.portalnepas.org.br/abcshs/article/ view/1006 